\title{
Optics experimental unit and analysis housing for maximum dielectrophoresis (DEP) and $\mathrm{AC}$ electrokinetics operations
}

\begin{abstract}
We have developed a straightforward optics system for lab on chip applications. This system can be used to handle a continuous micro fluid flow, submicron and nano-sized particles. The device that is investigated under this system can be used to fields such as bioseparation, biosensors for diagnostic and prognostic purposes. In our advance, the system is formed for dielectrophoretic (DEP) colloidal particle deflection application. Such device has been fabricated using glass substrates with microelectrode arrays on it and a microchannel made from Dry Film Resist (DFR). The fabrication ensures the easiness of its usage under the experimental unit. The system introduced can reduce cost, sophisticated hardware usage and power consumption. Separation via deflection (DEP) method was performed using test particles over a wide range of applied field frequencies, confirming the good deflection within the optics experimental unit setup.
\end{abstract}

Keyword: Lab on a chip (LoC); Dielectrophoresis (DEP); Optics experimental setup 